\title{
Beiträge zur Frage der Feinstrukrur des Polarisationsspektrums photolumineszierender Farbstoffe
}

\author{
Von N. MAREK \\ Institut für Medizinische Chemie der Medizinischen Universität Szeged, Ungarn \\ (Z. Naturforschg. 19 a, 371-374 [1964] ; eingegangen am 4. September 1963)
}

\begin{abstract}
Unter Verwendung einer geeigneteren Definition des wahren Polarisationsgrades der Lumineszenz wird der auf das langwellige Gebiet des Absorptionsspektrums entfallende Bereich des Polarisationsspektrums eingehend untersucht. In Verbindung mit der hier erstmalig gezeigten Vibrationsstruktur des Polarisationsspektrums wird auf einige neuere Möglichkeiten der Molekülstrukturforschung hingewiesen. Unsere theoretischen Überlegungen suchten wir an Hand von Messungen an hochviskosen Lösungen organischer Moleküle vom Typ des Acridins, Xanthens und Anthracens zu beweisen.
\end{abstract}

Bisher hat man, wie den übrigen Elektronenübergängen des Absorptionsspektrums, auch der langwelligen Bande einen einzigen Wert für den Polarisationsgrad zugeordnet. Diese Ansicht wurde auch von uns vertreten ${ }^{1}$, denn nach experimentellen Ergebnissen von Feofilow ${ }^{2}$ schien es, daß unter gewissen Versuchsbedingungen der $p(\lambda)$-Wert auch im Gebiet dieses Elektronenüberganges einem einzigen Grenzwert zustreben würde, wenn man die depolarisierende Wirkung der sekundären Fluoreszenz ${ }^{3}$ und der Konzentrationsdepolarisation berücksichtigt. Auf diese Weise gelang es auch, das von den Versuchsbedingungen unabhängige Polarisationsspektrum durch die Funktion

$$
P(\lambda, \eta)=\lim _{\tau \rightarrow 0} p^{\prime}(\lambda, \eta)
$$

darzustellen.

Diese Funktion ersetzt dabei die Grenzfunktion

$$
p_{0}(\lambda)=\lim _{\substack{c \rightarrow 0 \\ \eta \rightarrow \infty}} p(\lambda, c, \eta),
$$

die nur mittels einer schwierigen Extrapolation bestimmbar ist. $P(\lambda, \eta)$ nimmt dabei auf Grund der Perrin-Lewschinschen Beziehung

$$
\frac{1}{p}=\frac{1}{p_{0}}+\left[\frac{1}{p_{0}}-\frac{1}{3}\right] \cdot \frac{k T}{v \eta}+A c \quad \text { mit } c=0
$$

die folgende Form an:

$$
P(\lambda, \eta)=p^{\prime}(\lambda, \eta) \frac{1+K / \eta}{1+\frac{1}{3}(K / \eta) \cdot p^{\prime}(\lambda, \eta)} .
$$

Dabei ist $K=(k T / v) \tau$. Ferner ist $p_{0}=p_{0}(\lambda), p=$ $=p(\lambda, c, \eta), k$ die Boltzmannsche Konstante, $T$ die

1 I. Ketskeméty, N. Marek u. B. SÁrkány, Acta Phys. et Chem., Szeged 4, 21 [1958].

2 P. P. Fefilow, Usp. Fiz. Nauk 36, 417 [1948]. absolute Temperatur, $v$ das Volumen des Moleküls des fluoreszierenden Stoffes, $\eta$ die dynamische Viskosität der Lösung, $\tau$ die Abklingzeit der Fluoreszenz, $A$ eine Konstante und $c$ die Konzentration.

Der Wert der in Formel (1) angegebenen Funktion, die im folgenden als Polarisationsspektrum betrachtet wird, läßt sich bei einer Anregungswellenlänge $\lambda_{i}$ aus den an zwei Lösungen - von der Viskosität $\eta_{1}$ und $\eta_{2}-$ gemessenen Werten $\mathrm{p}_{i 1}$ und $\mathrm{p}_{i 2}$ bestimmen.

Nach Überwindung apparativer Schwierigkeiten, die der Untersuchung der Feinstruktur der Polarisationsspektren bis jetzt im Wege standen, zeigen die vorliegenden Experimente nun aber, daß der Polarisationsgrad des Fluoreszenzlichtes im Bereich der langwelligen Absorptionsbanden von der Wellenlänge des erregenden Lichtes abhängt und die Form einer Treppenfunktion besitzt. Dieser treppenartige Verlauf des Polarisationsspektrums entlang der kurzwelligen Seite der langwelligsten Absorptionsbande ist für die untersuchten Substanzen in Abb. 2 bis 5 dargestellt. Daneben sind das Absorptionsspektrum und das Emissionsspektrum im Bereich der reinen Elektronenübergänge aufgetragen. Bekanntlich entspricht das Polarisationsspektrum im Gebiet je eines Elektronenüberganges gewöhnlich einem konstanten $p$-Wert. Andererseits macht die Erfüllung der Spiegelsymmetrie, z. B. in der Darstellungsweise von Lewschin (Abb. 1), die Existenz mehrerer Elektronenübergänge in solch einem Bereich nicht wahrscheinlich. Die trotzdem gefundene Strukturierung des Polarisationsspektrums wird daher folgendermaßen erklärt:

3 Á. Budó, I. Ketskeméty, E. Salkovits u. L. Gargya, Acta Phys. Hung. 8, 181 [1957]. 
Nach Pringsheim ${ }^{4}$ kann für die Interpretation der Spektren von vielatomigen Molekülen in Lösung bei Erfüllung der Spiegelsymmetrie für das Absorptions- und Emissionsspektrum das Schwingungstermsystem der gelösten Moleküle durch das eines zweiatomigen Moleküls ersetzt werden, dessen Vibrationsfrequenz und Anharmonizität im angeregten $\mathrm{Zu}$ stand ebenso groß ist wie im Grundzustand. Diese Vibrationsfrequenz $\omega$ („prädominante Frequenz“ nach Pringsheim) verursacht bei Benzol und Anthracen z. B. die Strukturierung des Absorptionsspektrums.

Nach unseren vorläufigen Untersuchungen am Anthracen entspricht die Struktur des Polarisationsspektrums den im Absorptionsspektrum auftretenden Schwingungsteilbanden und auch der aus dem RamanSpektrum bestimmten totalsymmetrischen Schwingung des Moleküls von $1400 \mathrm{~cm}^{-15}$. Bei Erfüllung der Spiegelsymmetrie kann also die Struktur von $p(\lambda)$ durch die Annahme erklärt werden, daß der Polarisationsgrad nicht im Bereich einer ganzen Elektronenbande, sondern nur im Gebiet je einer Teilbande konstant ist.

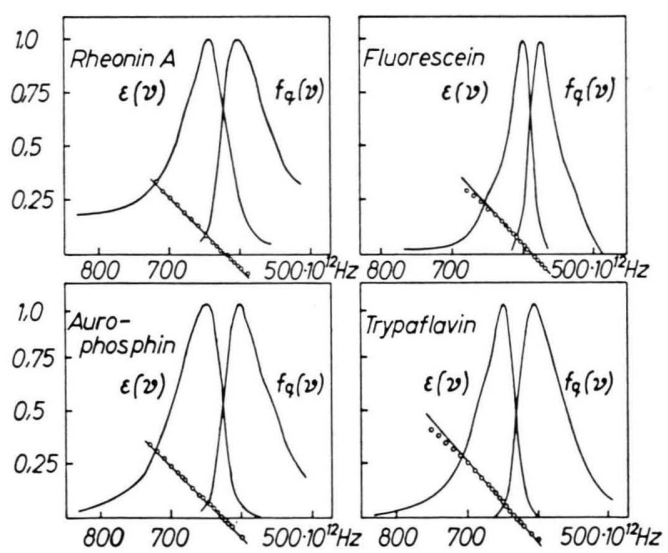

Abb. 1. Die Spiegelsymmetrie der Absorptions- und Emissionsspektren von Trypaflavin, Aurophosphin, Rheonin A und Fluorescein.

Dies gilt auch für fluoreszierende Stoffe, die nicht strukturierte Absorptions- und Emissionsspektren ihrer Lösungen zeigen, bei denen also die Schwingungsteilbanden infolge relativ großer Bandenbreite verschwimmen.

4 P. Pringsheim, Fluorescence and Phosphorescence, Interscience Publishers, Inc., New York 1949.

\section{Substanzen und experimentelle Methoden}

Untersucht wurden die Acridinfarbstoffe Trypaflavin, Aurophosphin und Rheonin A und der Xanthenfarbstoff Fluorescein. Bei der Messung der Absorptionsspektren wurden im Falle der Acridinderivate äthylalkoholische Lösungen von der Konzentration $3 \cdot 10^{-4}$ $\mathrm{Mol} / l$ benutzt. Die so gewonnenen Absorptionsspektren stimmen hinsichtlich der Form mit denen der glycerinäthylalkoholischen Lösungen der betreffenden Farbstoffe überein und können deshalb mit den Polarisationsspektren verglichen werden. Die Absorption des Fluorescein wurde an einer $10^{-4}$-mol. Lösung gemessen, mit einem Gemisch aus Glycerin (96,6 Vol.-Proz.), Wasser und $\mathrm{NaOH}$ (1 Gew.-Proz.) als Lösungsmittel. Die Daten bezüglich der Konzentration der Lösungsmittel, der Schichtdicke und der Viskositätsverhältnisse der bei den Bestimmungen der Polarisationsspektren benutzten Lösungen sind in Tab. 1 zusammengefaßt. Die sekundäre Fluoreszenz wurde durch entsprechende Wahl der Schichtdicken eliminiert.

\begin{tabular}{|c|c|c|c|c|}
\hline & $\begin{array}{c}c \\
\mathrm{Mol} / l\end{array}$ & Lösungsm. & $\begin{array}{c}l \\
\mathrm{~cm}\end{array}$ & $\begin{array}{c}\eta_{30^{\circ}} \\
\mathrm{cP}\end{array}$ \\
\hline Trypaflavin & $9 \cdot 10^{-5}$ & $\begin{array}{l}95,5 \% \text { Gl. } \\
+\mathrm{C}_{2} \mathrm{H}_{5} \mathrm{OH} \\
75 \% \text { Gl. } \\
+\mathrm{C}_{2} \mathrm{H}_{5} \mathrm{OH}\end{array}$ & 0,193 & $\begin{array}{r}434,82 \\
96,43\end{array}$ \\
\hline $\begin{array}{l}\text { Auro- } \\
\text { phosphin }\end{array}$ & $9 \cdot 10^{-5}$ & $\begin{array}{l}91 \% \text { Gl. } \\
+\mathrm{C}_{2} \mathrm{H}_{5} \mathrm{OH} \\
60 \% \text { Gl. } \\
+\mathrm{C}_{2} \mathrm{H}_{5} \mathrm{OH}\end{array}$ & 0,193 & $\begin{array}{r}326,72 \\
34,09\end{array}$ \\
\hline Rheonin A & $9 \cdot 10^{-5}$ & $\begin{array}{l}91 \% \mathrm{Gl} . \\
+\mathrm{C}_{2} \mathrm{H}_{5} \mathrm{OH} \\
71 \% \mathrm{Gl} . \\
+\mathrm{C}_{2} \mathrm{H}_{5} \mathrm{OH}\end{array}$ & 0,193 & $\begin{array}{r}306,36 \\
98,17\end{array}$ \\
\hline Fluorescein & $10^{-4}$ & $\begin{array}{l}96,62 \% \mathrm{Gl} . \\
1 \% \mathrm{NaOH} \\
80 \% \mathrm{Gl} . \\
1 \% \mathrm{NaOH}\end{array}$ & $\begin{array}{l}0,193 \\
0,0454 \\
0,0156\end{array}$ & $\begin{array}{r}436,94 \\
62,75\end{array}$ \\
\hline
\end{tabular}

Tab. 1.

Die Absorptionsspektren wurden mit dem Gitterspektrophotometer Optica Milano „CF 4“ aufgenommen. Zur Messung der Polarisationsspektren wurde im wesentlichen die von KETSKEMÉTY und SZALAY ${ }^{6}$ beschriebene Meßeinrichtung verwendet, nur erfolgte die Beobachtung in longitudinaler statt in transversaler Richtung. Zur Herstellung des monochromatischen Erregungslichtes diente ein Doppelmonochromator, zusammengesetzt aus einem $\mathrm{Z}$ e is s-Spiegelmonochromator und einem $\mathrm{Hilger-Prismenmonochromator.} \mathrm{Als} \mathrm{ge-}$ kreuztes Filter wurden S c h o t t - Glasfilter GG 14 bzw. OG 4 benützt.

5 G. Kortüм u. B. Finckh, Z. Phys. Chem. B. 52, 263 [1942].

6 I. Ketskeméty u. L. Szalay, Acta Phys. Hung. 5, 305 [1955]. 
Die Aufnahme der Emissionsspektren erfolgte nach der von Ketsкeméty ${ }^{\boldsymbol{}}$ beschriebenen Methode mit dem entsprechend ergänzten Gitterspektrophotometer $\mathrm{O} p$ ti c a Milano „CF 4“. Es wurden alkoholische Lösungen verwendet im Falle des Trypaflavins $\left(c=3 \cdot 10^{-4}\right.$ $\mathrm{m} / l, \quad d=0,6335 \mathrm{~cm})$, Aurophosphins und Rheonin A $\left(c=9 \cdot 10^{-5} \mathrm{~m} / l, d=1,005\right)$. Fluorescein wurde in einer Lösung aus 95 Vol.-Proz. Glycerin-Wasser +1 Gew.-Proz. $\mathrm{NaOH}$ bei einer Konzentration von $5 \cdot 10^{-4} \mathrm{~m} / l$ gemessen.

\section{Auswertung der experimentellen Ergebnisse}

Bei den in Tab. 2 zusammengefaßten Meßergebnissen sind die in Spalte 4 befindlichen Werte mit Hilfe des spiegelsymmetrischen Zusammenhangs bzw. der reinen Elektronenübergangsfrequenz $v_{\mathrm{el}}$ festgestellte Wahrscheinlichkeitswerte, da die Messungen, wegen der im Überlappungsgebiet durch die longitudinale Beobachtung auftretenden Schwierigkeiten, nur bis zum Absorptionsmaximum durchgeführt wurden. Wenn nach Festlegung des reinen Elektronenüberganges bei der Bestimmung der kurzwelligsten Vibrationsteilbande auf Grund des Verlaufs des Polarisationsspektrums Zweifel auftauchen, z. B. im Falle des Rheonin A, so kann die fragliche Vibrationsbande auf Grund der mittels Kombination der Polarisationsstufen berechnetenWinkelabweichungen zwischen Emissions- und Absorptionsoszillator bestimmt werden, die infolge der Vibration zustande kommen.

Nach Tab. 2 ist im Falle der drei Acridinfarbstoffe eine angenäherte Übereinstimmung der Vibrationsfrequenzen festzustellen.

Der unterschiedliche Abstand der Schwingungsbanden von Heteroatome enthaltenden, substituierten Verbindungen kann mit der Überlagerung der Eigenfrequenzen der einzelnen Atomgruppen in Beziehung gebracht werden.

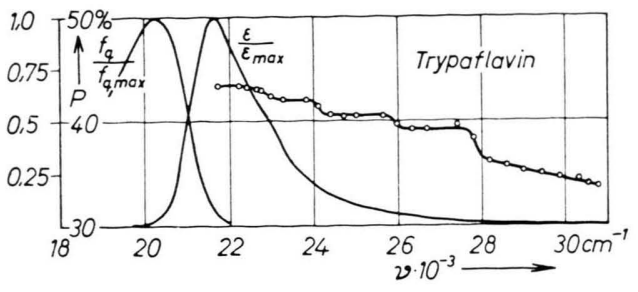

Abb. 2. Die langwellige Absorptionsbande des Trypaflavins -; die Vibrationsstruktur des Polarisationsspektrums von Trypaflavin $\mathrm{O}-\mathrm{O}-\mathrm{O}$.

Somit scheint die Wirkung eines neuen Substituenten auf die ursprünglichen Eigenschaften des Moleküls einer Untersuchung zugänglich zu sein, wenn, bei Kenntnis der prädominanten Schwingungen des Moleküls, das Studium der Vibrationsfrequenzen eine Analyse der superponierenden Frequenzen erlaubt. Für derartige Untersuchungen empfiehlt sich vor allem das Anthracen und Stoffe mit ähnlich gut strukturierten Absorptionsspektren.

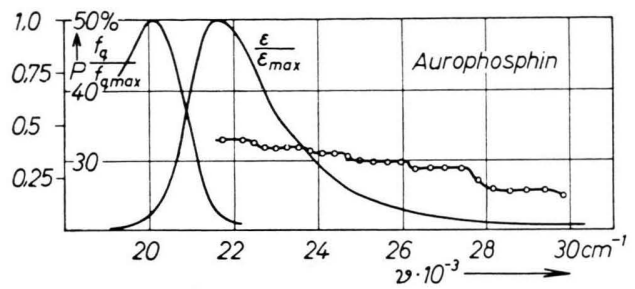

Abb. 3. Die langwellige Absorptionsbande des Aurophosphins -; die Vibrationsstruktur des Polarisationsspektrums von Aurophosphin o- $\mathrm{O}-\mathrm{O}$.

In Tab. 3 sind neben den auf Grund der obigen Überlegungen festgesetzten reinen Elektronenübergängen auch die an Hand des Polarisationsspektrums nachweisbaren Wellenlängen der kurzwelligsten Vibrationsteilbanden mit den ihnen zugehörigen $p$-Werten eingetragen. Ebenso sind die diesen Polarisationsgraden entsprechenden, vom Absorptionsoszillator mit dem Emissionsoszillator eingeschlosse-

\begin{tabular}{|c|c|c|c|c|c|c|c|c|}
\hline \multirow[b]{2}{*}{ Trypaflavin } & \multicolumn{8}{|c|}{ Wellenzahl in $\mathrm{cm}^{-1}$ und Wellenlänge in $\mathrm{m} \mu$} \\
\hline & & $\begin{array}{r}21030 \\
\quad 475,5\end{array}$ & $\begin{array}{r}22026 \\
454\end{array}$ & & $\begin{array}{r}23474 \\
426\end{array}$ & $\begin{array}{r}25062 \\
399\end{array}$ & $\begin{array}{l}26991 \\
\quad 370,5\end{array}$ & $\begin{array}{r}29673 \\
337\end{array}$ \\
\hline Aurophosphin & & $\begin{array}{c}20898 \\
478,5\end{array}$ & $\begin{array}{r}21978 \\
455\end{array}$ & $\begin{array}{r}23148 \\
432\end{array}$ & $\begin{array}{r}24271 \\
412\end{array}$ & $\begin{array}{r}25477 \\
392,5\end{array}$ & $\begin{array}{r}26917 \\
371,5\end{array}$ & $\begin{array}{c}29200 \\
342,7\end{array}$ \\
\hline Rheonin A & & $\begin{array}{r}20876 \\
479\end{array}$ & $\begin{array}{r}21834 \\
458\end{array}$ & $\begin{array}{r}22779 \\
439\end{array}$ & $\begin{array}{r}23923 \\
418\end{array}$ & $\begin{array}{r}25380 \\
394\end{array}$ & $\begin{array}{c}26863 \\
372,2\end{array}$ & $\begin{array}{r}28169 \\
355\end{array}$ \\
\hline Fluorescein & 19660 & $\begin{array}{r}20408 \\
490\end{array}$ & $\begin{array}{r}21857 \\
\quad 457,5\end{array}$ & $\begin{array}{r}23121 \\
432,5\end{array}$ & $\begin{array}{r}24540 \\
407,5\end{array}$ & $\begin{array}{r}25974 \\
385\end{array}$ & & \\
\hline
\end{tabular}

Tab. 2.

7 I. KeтsкемÉty, Acta Phys. Hung. 10, 429 [1959]. 
nen Winkel $\alpha$, sowie deren Differenzen $\Delta \alpha$ dargestellt. Diese Winkeländerung kommt durch die Anregung der Vibrationsübergänge zustande.

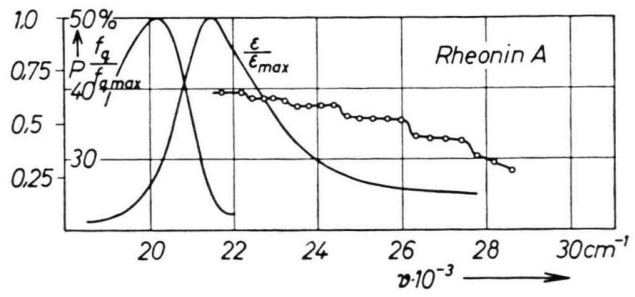

Abb. 4. Die langwellige Absorptionsbande des Rheonin A -; die Vibrationsstruktur des Polarisationsspektrums von Rheonin A O- $\mathrm{O}-\mathrm{O}$.

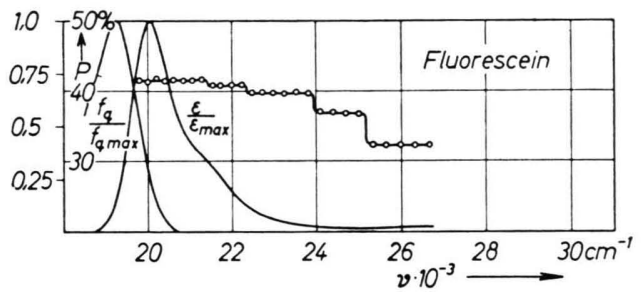

Abb. 5. Die langwellige Absorptionsbande des Fluoresceins ; die Vibrationsstruktur des Polarisationsspektrums von Fluorescein $\mathrm{O}-\mathrm{O}-\mathrm{O}$.

Der mit dem $\Delta \alpha$-Wert berechnete Polarisationsgrad steht auch trotz der mäßig gesteigerten depolarisierenden Wirkung der sekundären Fluoreszenz diese wurde durch geeignete Wahl der Schichtdicke auf unterhalb der Fehlergrenze liegende Werte herabgesetzt - im Gebiet der $v_{\mathrm{el}}$ in gutem Einklang mit dem Polarisationsgrad, der dem Schnittpunkt der Verlängerung der Schwerlinie unserer Meßpunkte mit der Geraden entspricht, die zur Frequenz $v_{\mathrm{em}}$ gehört $\left(v_{\mathrm{em}}=\right.$ Frequenz des beobachteten Fluoreszenzlichtes).

Eine ähnliche Grenzpolarisationsbestimmung hat Sewtschenko ${ }^{8}$ bei der Auswertung an der Seite des Emissionsspektrums angewandt. Selbstverständlich ist zur genauen Bestimmung der $\Delta \alpha$-Werte die exakte
Feststellung der kurzwelligen Grenzen der Vibrationsstruktur unerläßlich. Die Ausarbeitung der entsprechenden Methode ist eine Aufgabe der Zukunft.

Durch die unter strengeren Versuchsbedingungen als bisher vorgenommene Messung des Polarisationsspektrums konnte also der innige Zusammenhang mit der Vibrationsstruktur der langwelligen Bande des Absorptionsspektrums organischer Moleküle nachgewiesen werden, die konjugierte Doppelbindungen enthalten. Dieser Zusammenhang wird um so wahrscheinlicher, als die obigen Untersuchungen an $\mathrm{zu}$ verschiedenen Typen gehörenden und auch hinsichtlich ihrer Ladung abweichenden organischen Molekülen - an dem Acridin-ähnlichen, kationartigen Trypaflavin, Aurophosphin und Rheonin A, sowie dem Xanthen-ähnlichen, anionartigen Fluorescein und dem elektro-neutralen Anthracen - durchgeführt wurden.

Im Falle einer Erfüllung des Gesetzes der Spiegelsymmetrie müßte unseres Erachtens auch an der Seite des Emissionsspektrums eine ähnliche spiegelsymmetrische strukturierte Veränderung des Polarisationsgrades erhalten werden. Sowohl die experimentelle Weiterentwicklung unserer Methode als auch ihre theoretische Verfeinerung wäre also überaus wünschenswert.

Über den Zusammenhang der kurzwelligen Banden von Absorptions- und Polarisationsspektren soll in einer späteren Arbeit berichtet werden.

Herrn Prof. Á. Budó, Direktor des Institutes für Experimentelle Physik der Universität Szeged, und Herrn Dozenten I. KeтsкемÉty ist der Verfasser für ihr stetes Interesse am Thema während der in ihrem Institut durchgeführten Messungen, Herrn Prof. A. KrámLI, Direktor des Institutes für Medizinische Chemie der Medizinischen Universität Szeged, für seine Hilfe bei der Publikation der Arbeiten zu aufrichtigem Dank verpflichtet, während er Herrn Prof. TH. Förster für seine gelegentlich der Durchsicht des Manuskriptes gemachten wertvollen Bemerkungen besonders dankbar ist.

\begin{tabular}{|c|c|c|c|c|c|c|c|c|}
\hline & \multicolumn{2}{|c|}{ Trypaflavin } & \multicolumn{2}{|c|}{ Aurophosphin } & \multicolumn{2}{|c|}{ Rheonin A } & \multicolumn{2}{|c|}{ Fluorescein } \\
\hline$i$ & 1 & 2 & 1 & 2 & 1 & 2 & 1 & 2 \\
\hline$\lambda_{i}$ & 337,5 & 460 & 345 & 460 & 355 & 460 & 380 & 505 \\
\hline$P_{i}$ & 35,08 & 43,36 & 25,40 & 32,81 & 30,00 & 39,41 & 31,14 & 41,5 \\
\hline$\alpha_{i}$ & $28,33^{\circ}$ & $18,65^{\circ}$ & $36,77^{\circ}$ & $30,26^{\circ}$ & $32,97^{\circ}$ & $23,77^{\circ}$ & $31,99^{\circ}$ & $21,26^{\circ}$ \\
\hline$\Delta \alpha$ & \multicolumn{2}{|c|}{$9,68^{\circ}$} & \multicolumn{2}{|c|}{$6,51^{\circ}$} & \multicolumn{2}{|c|}{$9,20^{\circ}$} & \multicolumn{2}{|c|}{$10,73^{\circ}$} \\
\hline
\end{tabular}

Tab. 3.

8 A. N. Sewtschenko u. G. P. Gurinowitsch, Dokl. Akad. Nauk SSSR 126, 979 [1959]. 